\title{
Computed tomography appearances of focal and diffuse hepatic disease in children
}



\section{Abstract}

Paediatric hepatic masses present a diagnostic challenge to physician and surgeon alike. Dynamic new scanning techniques such as spiral computed tomography (CT) and current real time ultrasound have revolutionised scanning of liver masses, while magnetic resonance imaging (MRI) has added a fresh new spin on this topic. Focal processes include congenital lesions such as choledochal and simple cysts, infective conditions like abscesses and hydatid cysts, and neoplasms. The commonest neoplasms include benign tumours such as haemangioma and haemangioendothelioma, and malignant tumours such as metastases, hepatoblastoma (HB) and hepatocellular carcinoma (HCC). Important diffuse processes include biliary atresia, fatty infiltration, cirrhosis, iron deposition and Budd Chiari syndrome. Cognisance should also be taken of focal lesions such as focal fatty change, infarcts, anatomic variants and extrahepatic masses, which may mimic hepatic masses. This article aims to demonstrate the key CT imaging features that help in identifying these conditions.

\section{Key words}

Liver abscess,

haemangioendothelioma, hepatoblastoma, hepatocellular carcinoma

\section{Introduction}

Paediatric hepatic masses, though uncommon, have received much attention in the literature. Their diverse histological spectrum and perplexing pathogenesis have precluded an entirely satisfactory classification, while recent advances in tumour genetics have added a new and exciting perspective to a complicated subject., ${ }^{1,2}$ As with any paediatric abdominal mass, the extensive differential diagnosis is best approached with a knowledge of the patient's age, clinical findings, laboratory data and imaging characteristics. Although ultrasonography remains the initial investigation of choice in the child with an abdominal mass, spiral CT, with its ability to provide dynamic, multi-phase scanning through rapid volumetric data acquisition, has revolutionised the imaging of hepatic masses. ${ }^{3}$ Although 


\section{from page 23}

many lesions share overlapping radiological features, analysis of the imaging characteristics, considered in conjunction with clinical data, allows the formulation of a narrow differential, and in some cases a specific, diagnosis. ${ }^{1}$ This pictorial review aims to demonstrate the key CT features of the more common paediatric hepatic masses.

\section{Focal liver disease}

\section{Congenital}

\section{Choledochal cysts and Caroli's disease}

Choledochal cysts are thought to arise secondary to reflux of pancreatic secretions into the biliary tree. Five types are classified depending on the distribution of biliary dilation. Extra-hepatic types present with a dilated common bile duct, which may extend to the porta hepatis and proximal hepatic parenchyma. In Caroli's disease, the intra-hepatic ducts are dilated. CT scans show multiple hypodense round or ovoid structures, with or without a branching, tubular configuration (Figure 1). An occasional

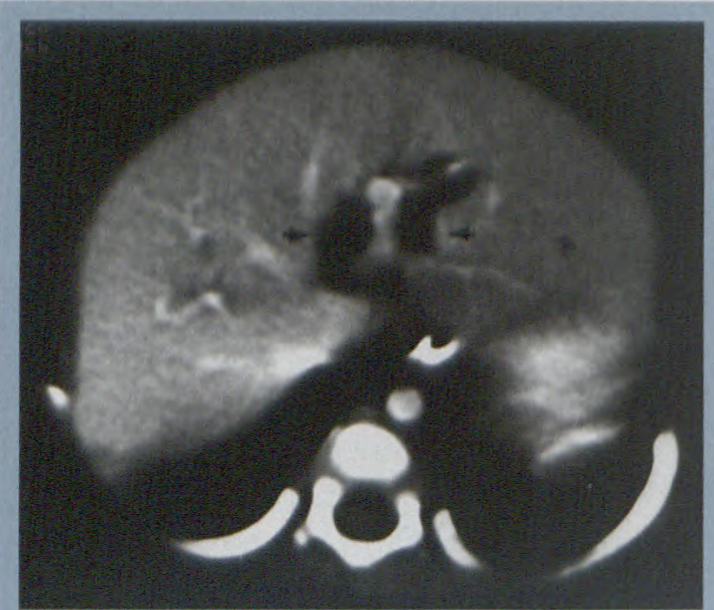

Figure 1: CT scan showing dillated central bile ducts (arrows) in a patient with Caroli's disease

enhancing focus may be seen, which represents a portal vein radical. ${ }^{1,4}$

\section{Cysts}

These are simple cysts that arise from the bile duct epithelium and are rare in children. They may be solitary or multiple and are usually unilocular, although septae may occasionally be present. The hepatic parenchyma is normal, distinguishing these from the simple cysts of polycystic hepatic disease in which there is hepatic fibrosis. On CT the cysts are well defined, round hypodense, non-enhancing lesions with occasional septae. ${ }^{1,3}$

\section{Infection}

\section{Pyogenic abscess}

Pyogenic organisms may enter the liver via the biliary tree (ascending cholangitis), by direct implantation (trauma, surgery) or by haematogenous spread (arterial, portal). In the latter instance, there is a predilection for the superior aspect of the right lobe. ${ }^{1,5}$ The typical CT appearance of a hypodense, rim-enhancing lesion is seen in $50 \%$ of cases, but features may vary depending on the viscosity of contents, haemorrhage and intralesional air (secondary to gas-forming organisms) (Figure 2). The "double target" sign seen on dynamic scans is suggestive of an abscess and consists of a lesion with a low-density centre, hyperdense rim and hypodense periphery. ${ }^{1}$ Appearances are, however, not specific and may also be seen with nonpyogenic abscesses or tumours.

\section{Granulomatous abscesses}

TB commonly causes nonspecific hepatomegaly owing to diffuse fatty infiltration, but visceral infection may result in multiple granulomatous abscesses that en- hance after contrast administration. Calcification occurs with healing, but does not imply quiescence of the lesion. TB is endemic in the Western

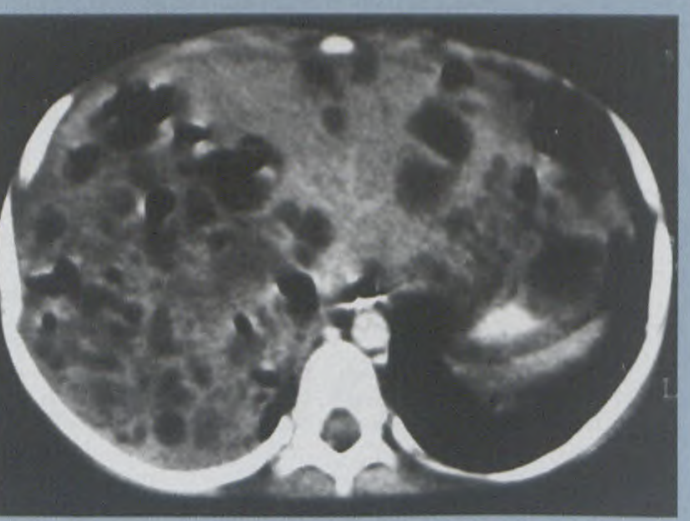

Figure 2: Contrasted CT of a child with multiple hepatic bscesses. Lesions are hypodense with rim enhancement. Airfluid levels indicate infection with gas-forming organisms.
Cape and hepatic tuberculous abscesses occur more frequently in the immunocompetent than immunocompromised patient. The burgeoning AIDS pandemic and growing number of organ transplant patients have seen a steady increase in the incidence of hepatic opportunistic infection, most commonly caused by organisms like Candida, Aspergillus, atypical mycobacteria and Pneumocystis. ${ }^{3,5}$ CT shows these microabscesses as multiple, small, round, hypodense lesions in the liver, and often also in the spleen (Figure $3)$. Occasionally large single or multiple fungal abscesses may resemble a pyogenic abscess. ${ }^{1,3}$

\section{Parasitic infestation}

Amoebic liver abscesses occur after colonic infection in approximately $8 \%$ of cases. $^{3}$ Appearances are often indistinguishable from pyogenic abscesses. ${ }^{3,5}$ Echinococcal cysts, most commonly caused by E. granulosus, consist of a three-layered wall: an outer pericyst, middle laminated layer and inner germinal layer. Patients are 


\section{from page 24}

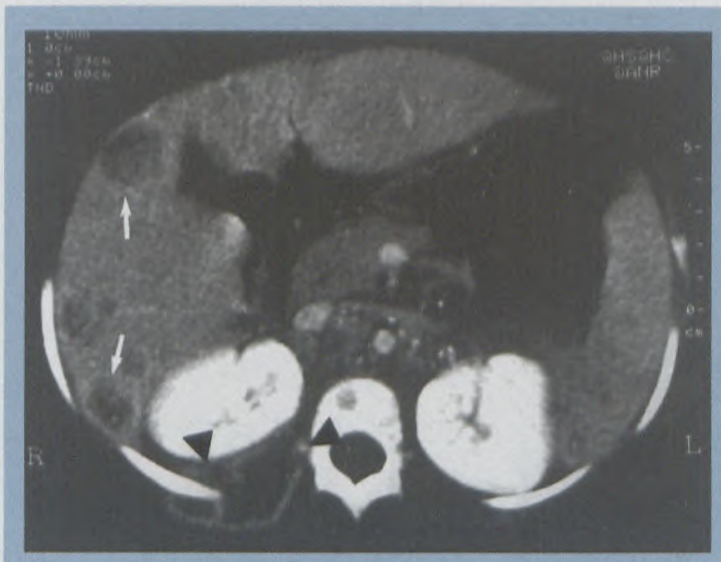

Figure 3: Contrast-enhanced scan of a patient with abdominal tuberculosis. The hepatic abscesses are visible as hypodense lesions with slight rim enhancement (arrows). There is an associated right psoas abscess (arrowheads).

usually asymptomatic until a cyst leaks or ruptures, when they present with fever, biliary colic, right upper quadrant (RUQ) pain or anaphylaxis. Ultrasonographically they present as solitary or multiple unilocular cysts or as a multilocular cyst with numerous daughter cysts, calcified internal membranes and hydatid sand. ${ }^{1,3,5}$ At $\mathrm{CT}$, the cysts typically measure fluid density, but may be complicated by haemorrhage and infection. The internal septations may enhance post-contrast administration. The water-lily sign refers to a contained cyst rupture in which the germinal layer floats within the pericyst (Figure 4).

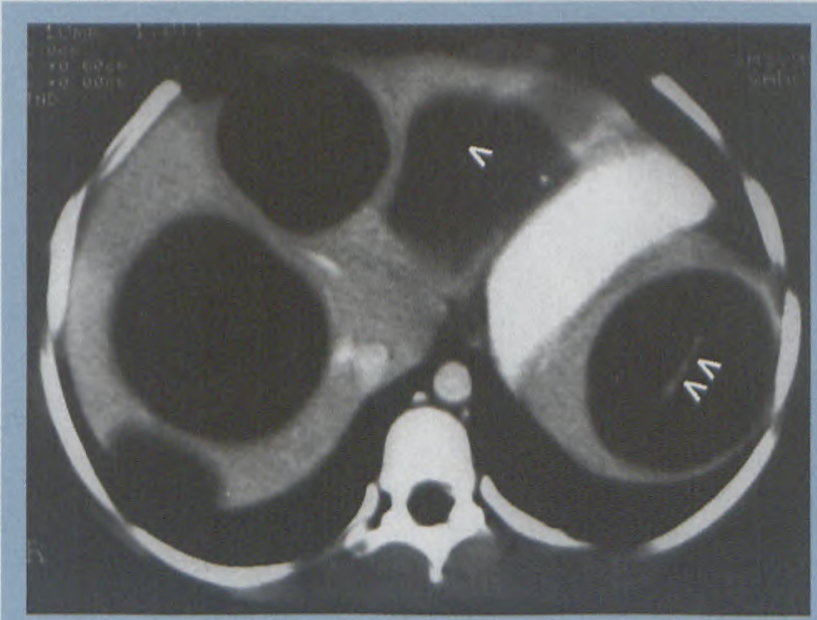

Figure 4: A patient with multiple hydatid cysts of the liver and spleen. The "water lily" sign (see text) is visible in the cysts in the spleen and left hepatic lobe (arrows).

\section{Neoplasms}

Hepatobiliary tumours represent about $6 \%$ of all paediatric tumours beyond the new-born period. Metastases are commoner than primary tumours. Primary hepatic malignancies are the third most common paediatric abdominal malignancy after neuroblastoma and Wilm's tumour. ${ }^{6}$ Two thirds of primary hepatic tumours are malignant and a third are benign. ${ }^{4}$

\section{Benign tumours}

\section{Haemangiomas}

Cavernous haemangiomas are the most common benign hepatic tumour and the second most common liver tumour overall (second only to metastases) ${ }^{3,5}$ They are usually seen in older children, may be single or multiple and classically occur in the posterior right lobe. The imaging features are described below.

\section{- Infantile haemangioendothelioma}

is a common neonatal tumour. More than $85 \%$ occur before the age of 6 months. ${ }^{1,6}$ Multiple histological subtypes occur, but the relation to adult types is unclear. Cavernous haemangiomas and haemangioendotheliomas have similar CT appearances. Precontrast scans show a hypodense, wellcircumscribed lesion with speckled calcification. Postcontrast rapid bolus dynamic scanning shows centripetal enhancement as flow occurs from peripheral to central sinusoids (Figure 5). During the arterial phase enhancing mural nodules are seen and probably represent vascular feeders. On delayed

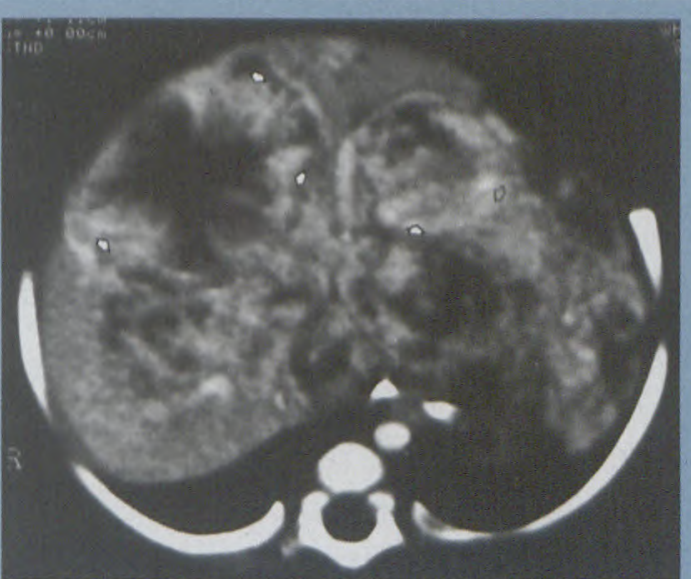

Figure 5: CT scan of infantile haemangioendothelioma shows multiple masses with typical lobulated peripheral enhancement (large arrows). The characteristic centripetal "filling in" by contrast is already evident in the tumour in the left lobe arlier scans not shown). scanning the lesion becomes isodense with hepatic parenchyma after 20-30 minutes. This typical pattern is seen in $54 \%$ of cases. $^{3}$ The remainder display absent, mixed or early central enhancement, indistinguishable from malignant lesions.

- Mesenchymal hamartomas. This rare entity, which consists of mixed mesenchymal and biliary tissue, is considered a developmental anomaly rather than a neoplasm. They usually present before the age of two years. At CT, the lesions are large, multiloculated, cystic masses similar in appearance to undifferentiated embryonal sarcoma (UES). ${ }^{6}$

- Lipomatous tumours. These are uncommon fatty tumours with varying proportions of adenomatous, angiomatous or myomatous stromal 


\section{from page 25}

elements. They usually occur in isolation, but have a recognised association with tuberous sclerosis. ${ }^{3}$ The CT appearance is generally that of a hypodense, well-circumscribed mass with variable enhancement proportional to the angiomatous or adenomatous elements. The differential diagnosis includes metastases from ovarian teratoma, fatty degeneration in an HCC and focal fatty change. ${ }^{3}$

\section{Malignant tumours}

\section{Metastases}

These are the most common hepatic tumours. The most important sources in children are Wilm's tumour, neuroblastoma, rhabdomyosarcoma and lymphoma. ${ }^{1} \mathrm{CT}$ appearances vary depending on the presence of tumour haemorrhage, necrosis, fibrosis or calcification. Most metastases present as multiple, well-circumscribed hypodense lesions. Calcification occurs with neuroblastoma secondary deposits. It has been stated that contrast enhanced scans add little to the sensitivity for tumour detection and that hypervascular metastases that become isodense to hepatic parenchyma may be missed if pre-contrast scans are not obtained. ${ }^{1,3}$ Dual and triple phase imaging has, however, added a new elegance to the demonstration of metastases. Metastases, such as Wilm's tumour, which have a predominantly hepatic arterial blood supply, usually enhance intensely in relation to the relatively unenhanced liver parenchyma on images obtained during the arterial phase of hepatic perfusion. During the portal venous phase, they may appear hypodense relative to the normally enhancing liver parenchyma (Figure 6). In contradistinction, hypovascular lesions are not usually

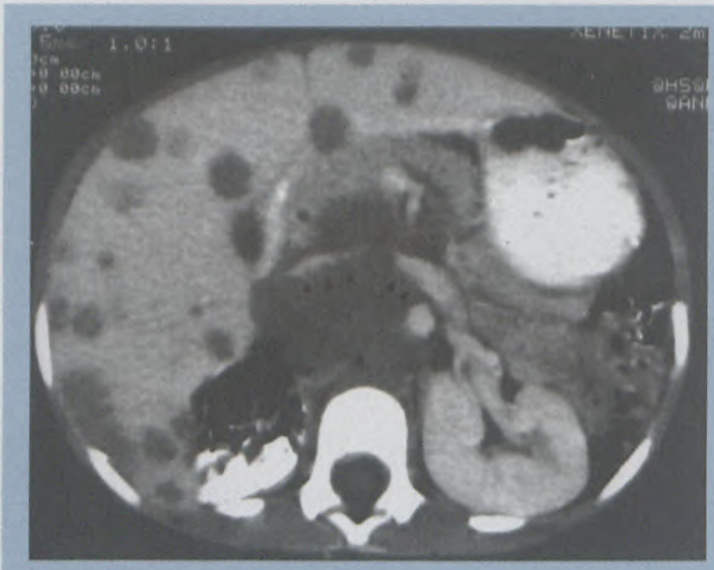

Figure 6: Contrast-enhanced CT scan of a patient with metastatic Wilm's tumour. The secondary deposits are visible as multiple, non-enhancing hypodense nodules. The IVC is displaced anteriorly and the left renal vein is draped across a large mass of lymph nodes (arrows).

visible on early images, but appear hypodense on scans during the portal venous phase. ${ }^{3}$

\section{Hepatoblastoma}

This is the third most common abdominal tumour and the most common primary malignant liver tumour in children. ${ }^{6}$ Most tumours occur in the first three years of life and there is a male preponderance. Important associations include BeckwithWeidemann syndrome, Gardner's syn-

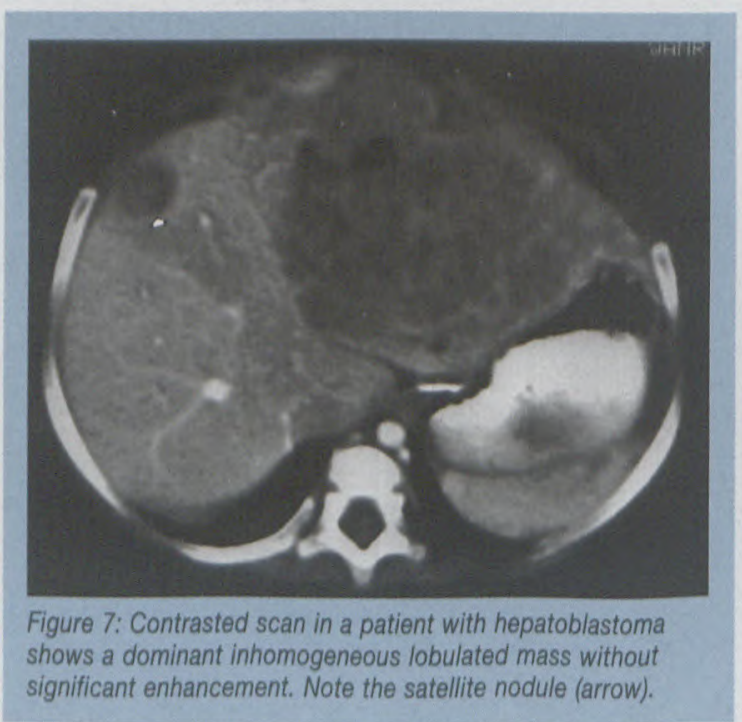

drome, Wilm's tumour and biliary atresia. ${ }^{6}$ Hepatoblastomas share a common chromosomal aberration with rhabdomyosarcoma and Wilm's tumour, viz. mutation at $11 \mathrm{p}$ locus. ${ }^{2}$
CT shows a heterogeneously hypodense mass with variable, peripheral enhancement. Calcification is present in up to $50 \%$ of cases and is coarse and nodular as opposed to the stippled pattern seen with haemangioendothelioma. ${ }^{1,3,5}$ Appearances may be indistinguishable from hepatoma (Figure 7).

\section{Hepatocellular carcinoma (HCC)}

$\mathrm{HCC}$ is the second most common primary malignant hepatic tumour in children. As with hepatoblastoma, there is a male preponderance, but most tumours occur after the age of five years. ${ }^{4} \mathrm{~A}$ pre-existing liver disease (e.g. cirrhosis, Wilson's disease, haemochromatosis, alpha-l antitrypsin deficiency, Gaucher's disease and chronic hepatitis) is present in half of the cases. ${ }^{3,6}$ Radiologically, HCC may take the form of a focal mass, multiple focal masses, a dominant mass with satellite nodules (Figure 8) or, less commonly, a diffusely infiltrating lesion. On CT, HCC may be hypo-, iso- or hyperdense depending on the internal composition (fatty areas, haemorrhage and calcification) and surrounding parenchyma (normal vs fatty change). Calcification occurs in up to $25 \%$ of tumours. ${ }^{1,3}$ Triple-phase scanning is particularly valuable - hepatomas are supplied by the hepatic artery and therefore become hyperdense to liver parenchyma in the arterial phase, hypodense during the portal phase and isodense on delayed scans. ${ }^{3}$ 


\section{from page 26}

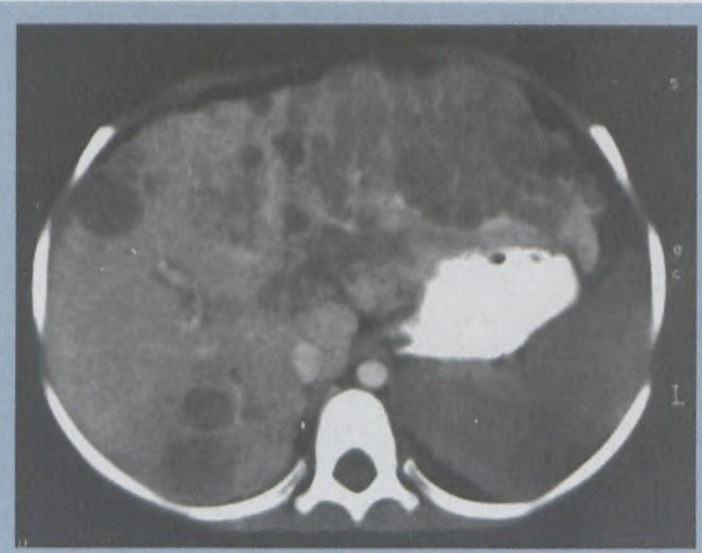

Figure 8: Similar appearance to that in Figure 7. This patient had hepatocellular carcinoma. The features are of a dominant inhomogeneous lobulated mass with irregular enhancement and numerous satellite nodules.

foci or peri-portal hypo-attenuation. ${ }^{6}$

Primary lymphoma, usually non-Hodgkin's lymphoma (NHL), commonly causes diffuse infiltration of the periportal tracts, with or without hepatomegaly. However, immunocompromised patients, especially those with AIDS, often develop multifocal hypodense nodules with variable enhancement, which must be differentiated from abscesses and

Septations, when present, enhance during the portal phase. Aorto-portal shunting may also be seen as early or prolonged portal vein enhancement. Venous involvement is visible either as enhancing tumour thrombus or non-enhancing bland thrombus., 3

\section{Lymphoproliferative disorders and lymphoma}

The incidence of lymphoproliferative disorder (LPD) is increasing owing to the growing number of AIDS and transplant patients. LPD is believed to be due to a proliferation of Epstein-Barr virus-infected cells and covers a spectrum of diseases from B-cell hyperplasia to monoclonal B-cell lymphoma. The CT scan shows scattered hypodense

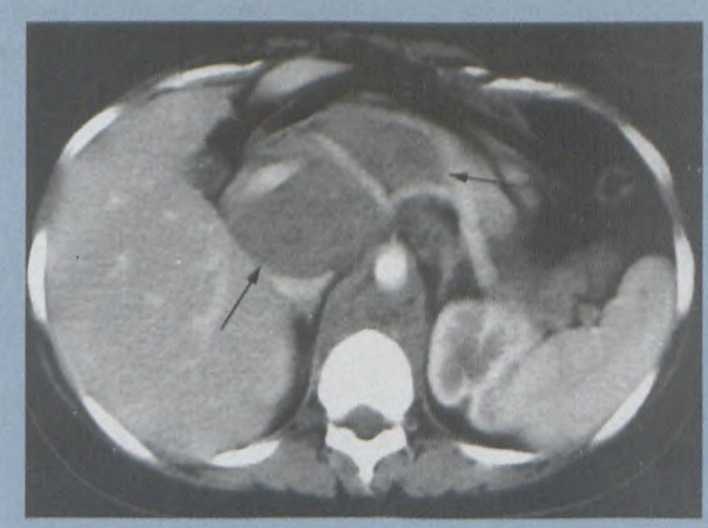

Figure 9: CT scan of a patient with Hodgkin's disease showing para-aortic lymphadenopathy (arrows). The liver was also infiltrated and enlarged. metastases, ${ }^{3,5}$ The liver is more commonly involved secondary to systemic lymphoma. The disease is usually diffusely infiltrating and causes hepatomegaly (Figure 9). Half of NHL and $60 \%$ of Hodgkin's disease cases involve the liver. ${ }^{3}$

\section{Sarcomas}

These are uncommon tumours. Undifferentiated (embryonal) sarcoma (UES), considered by some to be the malignant counterpart of the mesenchymal hamartoma, is the third most common primary paediatric hepatic malignancy. ${ }^{6}$ It constitutes $13 \%$ of hepatic tumours in children and usually occurs between 6 and 10 years of age ${ }^{2,6} \mathrm{On} \mathrm{CT}$ the tumour is largely cystic with variable solid components. Rim enhancement is occasionally present and calcification is more common than in embryonal rhabdomyosarcoma. ${ }^{1}$ The mesenchymal hamartoma may have an identical appearance to UES, but it occurs in a younger age group. ${ }^{6}$

Rhabdomyosarcoma of the biliary tree often occurs in the porta hepatis and presents with biliary obstruc- tion. CT reveals a well or poorly defined, hypodense mass that may be heterogeneously solid or nearly totally cystic. ${ }^{1,2}$ Other sarcomas are extremely rare.

\section{Diffuse disease}

\section{Fatty infiltration}

Fatty change may be focal, geographic or diffuse and, as such, may mimic primary or secondary tumours. Diffuse fatty infiltration is seen in malnourished patients, patients with chronic illness, e.g. TB, and oncology patients secondary to chemotherapy and hyperalimentation. ${ }^{5}$ Normal hepatic parenchyma is approximately 10 $\mathrm{HU}$ greater than the spleen on nonenhanced CT scans (NECT). In diffuse fatty infiltration this relationship is reversed and hepatic attenuation is typically $10 \mathrm{HU}$ less than that of the spleen on NECT and 25 HU less on enhanced scans (Figure 10), 5,6

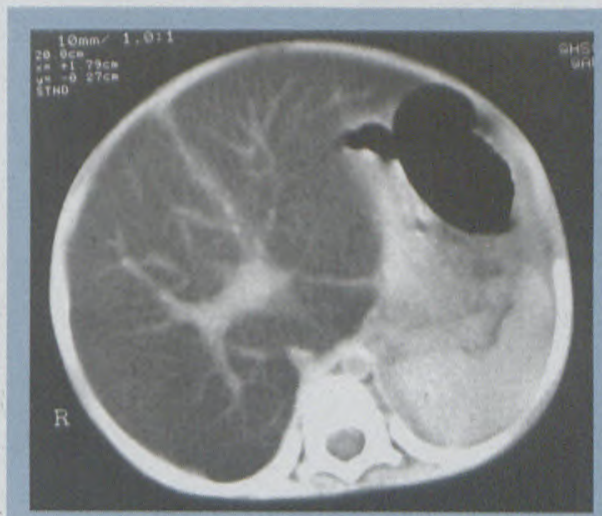

Figure 10: The liver is diffusely hypodense owing to fatty infiltration in this patient with TB. Note the relative hyperdensity of the spleen and hepatic vessels in the non-contrasted scan.

\section{Cirrhosis}

Paediatric causes include viral hepatitis, biliary atresia, cystic fibrosis, Alagille syndrome (arteriohepatic dysplasia), hereditary tyrosinaemia, alpha- 1 antitrypsin deficiency, congenital hepatic fibrosis, haemochromatosis and chronic congestion, e.g. Budd- 


\section{from page 27}

Chiari syndrome. CT typically shows an irregular contour with multiple hyper- or hypodense regenerating nodules, usually less than $2 \mathrm{~cm}$ in size, but that may occasionally attain sizes large enough to be palpable abdominal masses (Figures 11 and 12). ${ }^{1} \mathrm{Com}-$

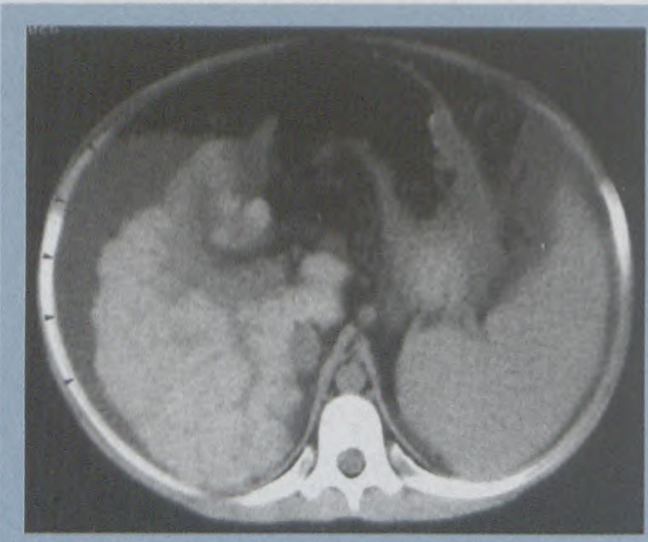

Figure 11: Uncontrasted scan in a patient with cirrhosis shows a shrunken liver and numerous regenerative nodules. Ascites also present (arrows).

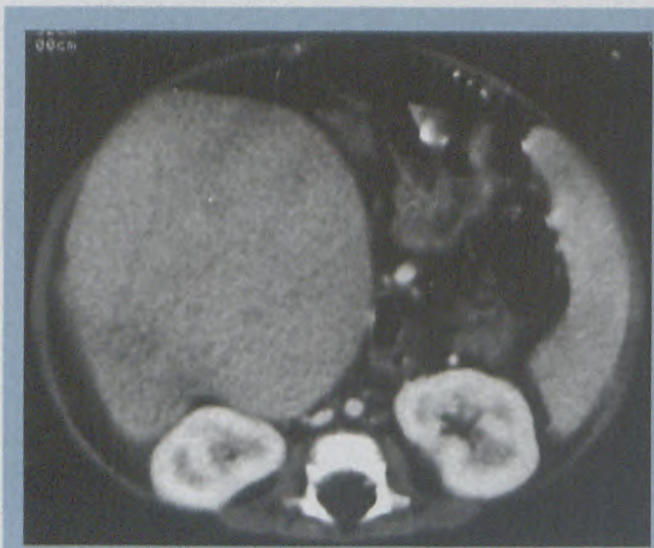

Figure 12: This scan demonstrates a large regenerative nodule, which was palpable abdominally, in a cirrhotic patient

plications such as fatty infiltration and HCC are also demonstrated at CT.

\section{Mineral deposition}

Iron deposition in haemochromatosis and transfusional haemosiderosis may cause progressive cirrhosis and occasionally $\mathrm{HCC}^{5}$ On pre-contrast CT the hepatic density is increased from $55-60 \mathrm{HU}$ to $70-100 \mathrm{HU}$. Iodine deposition in glycogen storage diseases also results in a hyperdense hepatic parenchyma.

\section{Budd-Chiari syndrome}

The condition is characterised by venous congestion secondary to hepatic vein obstruction. This may be idiopathic or secondary to tumour, congenital webs, phleboliths or thrombosis (tumour or blood dyscrasia). Microvenous occlusion may also occur in patients receiving chemo- or radiotherapy. At CT the liver is enlarged, with enhancement of the periportal parenchyma, including a hypertrophied caudate lobe. The peripheral parenchyma is hypoattenuating. ${ }^{5}$ The inferior vena cava and hepatic veins may be attenuated and prominent collaterals may be visible, paralleling the "spider web" appearance seen on angiography (Figure 13).

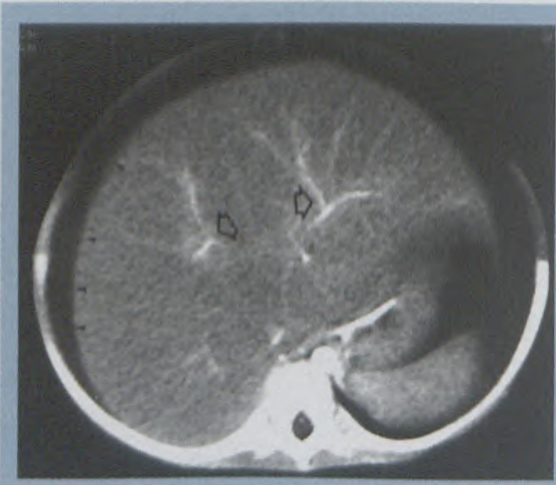

Figure 13: Contrasted scan in a patient with Budd-Chiari syndrome. The liver is enlarged and of decreased density. The IVC is attenuated and multiple enhancing collaterals are visible (arrows). Ascites also present (arrowheads).

\section{Amyloidosis}

This disease is characterised by the extracellular deposition of beta-pleated sheet protein fibrils and may be primary in origin or secondary to systemic illness. The liver is diffusely enlarged, but occasionally there may be hypodense nodules (Figure 14)

\section{Focal disease that may mimic masses}

\section{Anatomic variants}

Important examples include an accessory fissure that demarcates the

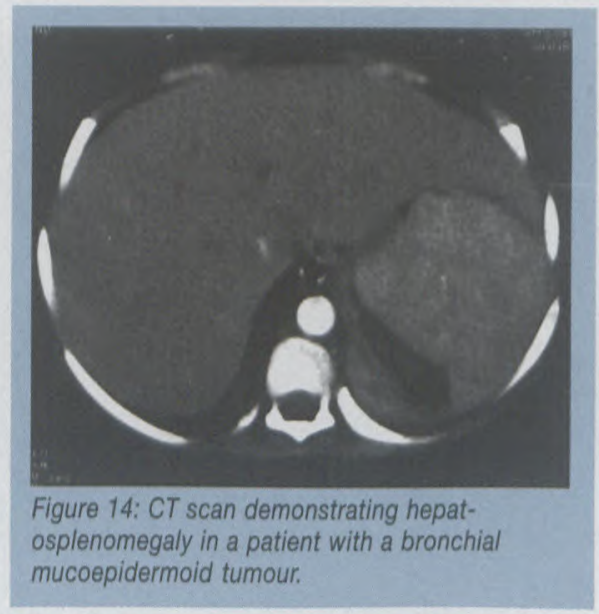

posterior segments of the right hepatic lobe and a Riedel's lobe.

\section{Focal fatty change}

This may be incorrectly interpreted as a hypodense mass, but the lesion's conforming to the hepatic contour without displacing vascular structures suggests the correct diagnosis. $^{1}$

\section{Iatrogenic}

An umbilical venous catheter used in neonatal intensive care units should have its tip situated in the superior aspect of the IVC between the diaphragm and the right atrium. Occasionally a malpositioned tip may lie in the right or left portal vein. A laceration with haematoma formation and thrombosis may occur and present as a mass. ${ }^{6}$

\section{Hepatic necrosis}

Necrosis may occur secondary to infection, trauma, toxins or infarction and mimic a hypodense mass. ${ }^{1}$

\section{Extrahepatic masses}

Wilm's tumour and neuroblastoma are notable examples of extrahepatic lesions, which, because of their position, may simulate intrahepatic masses.

\section{Conclusion}

Focal and diffuse hepatic disease has abroad spectrum of clinical presentations 